\title{
ALBERTO LISTA, DIRECTOR DE LA GACETA (1833): DOCUMENTOS
}

\author{
Diego MARTÍNEZ TORRÓN \\ Universidad de Córdoba
}

En el Archivo Histórico Nacional de Madrid, Sección Consejos, Serie Impresiones, legajo $11323 \mathrm{n}^{\circ} 16$, legajo $11331 \mathrm{n}^{\circ} 8$ y legajo $11331 \mathrm{n}^{\circ} 29$, se encuentran algunos documentos sobre el nombramiento de Alberto Lista como director de La Gaceta en 1833, entre ellos unas hojas nanuscritas de Lista informando sobre el personal de la publicación, y sobre la petición de sus libros a Francia.

Añado la transcripción a máquina de estos textos manuscritos a los más relevantes ya publicados en el Homenaje a Don Antonio Vilanova (Universidad de Barcelona) y en el Homenaje a Don Antonio Gallego Morell (Universidad de Granada), en espera de poderlos comentar más ampliamente en un libro que preparo desde hace tiempo sobre Alberto Lista'.

El citado legajo $11331 \mathrm{n}^{\mathrm{o}} 8$ contiene una carta de Pedro de la Hoz, remitida desde la imprenta, en la que se resume su asunto con las palabras: "renuncia del $\mathrm{p}^{\text {er }}$. redactor de la Gaceta" al margen. También al margen figura el siguiente texto, con otro letra: "12 de Enero de 1833. S.M. admite la renuncia dándose por satisfecho de sus servicios en el tpo. que la ha desempeñado; y ha nombrado en su lugar a Dn. Alberto Lista. Mes de enero día 12".

La carta de Pedro de la Hoz dice lo siguiente:

1. A estos trabajos habría que añadir los valiosos inéditos —epistolario y poemas—que publicará el $B R A E$ en su primer fascículo de 1991, y la Memoria sobre el feudalismo que publicó el $B R A H$ en tomo CLXXXVII, cuaderno II, pp. 299-319, 1990. 
Exmo. señor: Cuando en Enero de 1831 se dignó S.M. conferirme, además del destino de Director de la gaceta que entonces desempeñaba, la fiscalía general de Correos, a la cual está unida por ordenanza la Subdelegación de la Ymprenta real, supuse que era obligación muy superior a mis débiles fuerzas el servicio de los dos empleos reunidos. Así lo manifesté inmediatamente al Exmo. Señor Secretario de Estado y del Despacho Dn. Manuel Gonz $z^{z}$ Salmón, y en los mismos términos lo espuse a sus sucesores siempre que cualquiera ocurrencia del servicio daba a conocer prácticamente la incompatibilidad de los dos cargos que jamás han estado juntos en una misma persona; pero sea que en la mía se pusiera una confianza que sólo por el celo que me anima puedo merecer, sea que se aspirara a economizar un sueldo, ni dichas manifestaciones bastaron para que se nombrara otro Director, ni yo creí que podía insistir por entonces en ellas sin riesgo de que se me culpara de negligencia o de ingratitud.

Pasados ya dos años; terminada felizmente la enfermedad del Rey N.S. ocurrida en los momentos en que yo iba a renovar mis gestiones, y agravados los males crónicos que han deteriorado mi constitución física en tanto grado como V.E. habrá advertido a primera vista, no puedo menos de pedir a V.E.se sirva proponer a S.M. que si no hubiera algún obstáculo para ello se me exonere de la dirección de la Gaceta: cosa tanto más conveniente cuanto que la publicación de nuevos periódicos con los cuales hay que concordar lo que se diga en la Gaceta, y la premura con que actualmente se egecuta la redacción, hace cada día más incompatibles dos destinos, de los cuales cada uno es sobradamente pesado para cualquier hombre aunque tenga mejor salud y más capacidad que yo tengo.

Dios gue. a V.E. ms. as. Madrid 10 de Enero 1833

Exmo. Señor: (firma) Pedro de la Hoz.

El escrito va dirigido al Exmo. Sr. Conde de Heredia y de Ofalia, Sec $^{\text {rio }}$. de Estado y del despacho del Fomento gen ${ }^{1 .}$ del Reyno.

En el mismo legajo y número se resume: "1833. Se admite la dimisión del Director de la Gaceta a Dn. Pedro la Hoz y se nombra para el mismo cargo a D. Alberto Lista". Se contienen además estos dos escritos:

A Dn. Pedro de la Hoz. Madrid 12 de Enero de 1833.

El Rey N.S. se ha servido admitir la renuncia de la Dirección de la Gaceta, que hace V.S. en papel de $10 \mathrm{del}$ actual, en atención al quebranto de su salud; y S.M. ha tenido a bien darse por satisfecho del mérito y servicios de V.S. en el tiempo que ha desempeñado el mismo destino, el cual seguirá al cargo de V.S. hasta la presentación de su sucesor. De orden de S.M. lo digo a V.S. para su inteligencia, satisfacción y demás efectos. Dios $V^{a}$.

Traslado al Subdelegado de la Ymprenta Real para su inteligencia y efectos correspondientes.

El otro escrito: ${ }^{2}$

2. Este escrito, que fechan en 12 de enero de 1833, firmado por Ofalia, lo recogen Manuel Chaves, 
A Dn. Alberto Lista. Dha fha.

El Rey N.S. se ha dignado nombrar a VD. Director de la Redacción de la Gaceta, en lugar de Dn. Pedro de la Hoz, a quien S.M. ha tenido a bien admitir la renuncia que ha hecho del propio destino en atención al quebranto de su salud. De Rl. orden lo digo a Vd. para su inteligencia, satisfacción y demás efectos. Dios $\mathrm{V}^{\mathrm{a}}$.

Traslado al Subdelegado de la Ymprenta Real $p^{a}$. su inteligencia y efectos correspondientes.

En el mismo legajo y número se encuentra un escrito manuscrito de Lista con el siguiente texto:

Exmo. Sr. Ministro Secretario de Estado y del Despacho Universal del Fomento:

Exmo. Señor: He recibido hoy el oficio de V.E. en que se sirve anunciarme que S.M. se ha dignado nombrame director de la Redacción de la Gaceta, admitiendo la renuncia que D. Pedro de la Hoz ha hecho del propio destino por causa de su quebrantada salud.

$\mathrm{Y}$ en su contestación debo decir que acepto con acción de gracias este empleo, suplicando a V.E. se sirva de manifestar a S.M. mi profunda gratitud por este favor y por la confianza con que me honra, y el infatigable celo con que procuraré emplear mis débiles fuerzas en su servicio.

Dios guarde a V.E. muchos años. Yrún, Lazareto del Puente, 18 de enero de 1833. (Firmado:) Alberto Lista.

En el legajo $11323 n^{\circ} 16$ se recoge un escrito con membrete del Ministerio del Fomento General del Reino, firmado por el Conde de Ofalia, con añadidos y correciones, dirigido al Sr. Secretario del despacho de Hacienda. El texto es el siguiente:

Excmo. Sr: En la carta de que dirijo copia a V.E. me da noticia el Director de la Redacción de la Gaceta Dn. Alberto Lista de las obras históricas que han sido de su uso en las empresas literarias que desempeñó por encargo del Gobierno y de particulares, y hace venir de Francia, donde las tenía, y ruta de Irún y Vitoria, en atención a serles indispensables para llenar cumplidamente los deberes de tal Director; por lo cual, y también por que ninguna sabe que esté prohibida, pide que se temple algun tanto la severidad de las leyes sobre introducción de libros extrangeros. El actual destino de Lista, sus anteriores trabajos literarios, y la necesidad de tales libros para que la redacción de la Gaceta se verifique con el decoro y propiedad que deben resplandecer en este único periódico oficial, son cosas que no admiten duda,

Don Alberto Rodríguez de Lista, Sevilla, Tipografía El Mercantil Sevillano, 1912, pp. 39-40 y Hans Juretschke, Vida, obra y pensamiento A. L., Madrid, CSIC, 1951, pp. 154-155. Pero solamente esta breve comunicación. 
y corroboran los fundamentos en que estriba su pretensión. Por 10 mismo espero que V.E. se servirá acordar con S.M. que los libros referidos de Lista se manden precintar y sellar en la aduana de Vitoria con sugeción a su revisión en la de esta Corte y que se les exima del pago de Reales dros. mediante el objeto a que son usados. Dios guarde a V.E. muchos años. Madrid 7 de Febrero de 1833.

(Firma:) El Conde de Ofalia.

Otro igual al Sr. Secretario del despacho de Gracia y Justicia con este pie.

Por lo mismo espero que V.E. se servirá acordar con S.M. se prevenga al Revisor del ordinario diocesano en la aduana de esta Corte $\mathrm{q}^{\mathrm{c}}$. no ponga reparo en el pase de los libros de Lista en atención al objeto con que vienen destinados. Dios $V^{i}$

Otro igual al Sr. Presidente del Consejo Rl. con esta conclusión.

Por lo mismo se servirá V.E. prevenir al Revisor Real de la aduana de esta Corte que no ponga reparo en el pase de los libros de Lista en atención al objeto con que vienen destinados. Dios $\mathrm{V}^{\mathrm{a}}$.

En el mismo legajo y número se encuentra un escrito:

A Dn. Alberto Lista. Madrid, 27 de febrero de 1833.

Con fecha de ayer me dice el Sr. Secretario del Despacho de Gracia y Justicia lo siguiente.

En contestación - $h^{i a}$. - convenientes.

$\mathrm{Y}$ de la misma Rl. orden lo traslado a Vd. $\mathrm{p}^{\mathrm{a}}$. su gobierno.

Dios Va.

Y otro escrito en cuyo margen figura: "27 de Febrero de 1833. Dígase al interesado". El escrito en cuestión es el que sigue:

Exmo. señor.

En contestación a la Real Orden que en 24 del presente y en consecuencia del oficio de V.E. sobre que no se opusiese reparo al pase de los libros de Dn. Alberto Lista dirigí al Vicario Eclesiástico de Madrid para que se permitiese por el revisor del ordinario la entrada de aquéllos, me dice hoy lo que sigue.

"Exmo. Señor: He recibido la Real orden que V.E. me comunica con fecha 24 pero no la acompaña la lista de libros pertenecientes a Dn. Alberto Lista para remitirla al Sr. Cardenal Arzobispo a quien privativamente, como a todos los demás Sres. Obispos, corresponde, cesante la Inquisición, esta clase de negocios".

Lo que de Real Orden comunico a V.E. para su inteligencia y efectos convenientes. Dios guarde a V.E. muchos años. Madrid 26 de Febrero de 1833. (Firmado:) Fernández del Pino.

Este escrito va dirigido al Sr. Secretario del Despacho del Fomento. En el resumen del asunto figura: "Dn. Alberto Lista Director de la redacción de la Gaceta sobre introd ${ }^{\text {on }}$. de obras históricas de su propiedad que hace 
venir de Francia. Visto bueno 27 febrero 1833."”

Finalmente, con membrete de la Dirección de la Redacción de la Gaceta, legajo $11331 \mathrm{n}^{2} 29$, con la indicación de "Reservado" figura un texto manuscrito de Lista, con la peculiar disposición secuencial que estudiamos a propósito de Roger de Flor. Al margen escribió otra persona: "3 de Noviembre de 1833. Dése orden al Subdelegado de la Ymprenta Real $p^{\mathbf{a}}$. $q^{\mathrm{e}}$. envíe nota nominal de todos sus empleados con expres ${ }^{n}$. de sus sueldos y clases. Dése ésta previniendo que queden de cesantes Dn. José de Nieva y Dn. Eugenio Amor, con el sueldo que les corresponda por sus años de servicio. S.M. nombra a Dn. Mig'. Salvá por oficial $1^{\circ}$. en lugar de Amor. Sedano pasa a primer redactor, y se suprimer la plaza de $2^{\circ}$. Al portero se le aumen$\tan$ quinientos rl. al año."

El informe reservado de Lista dice lo que sigue:

Exmo. Señor: He recibido el oficio de V.E. de 25 de octubre, en que anuncia haber mandado hacer en París la suscripción al periódico inglés de Galignani para el uso de esta redación.

Yo renuevo la súplica que ya hice al antecesor de V.E., pidiéndole un oficial para esta redacción que entendiese el idioma inglés, pues entre los actuales no hay ninguno que lo sepa. Y propongo de nuevo para este destino a Dn. Miguel Salvá, individuo de la academia de la Historia, sacerdote de purísimas costumbres, muy instruido en lenguas y erudición, y cuyas opiniones son las que el gobierno debe desear en todos sus empleados.

Para que V.E. conozca bien la situación de esta oficina de mi cargo, le formaré el estado de alta. Consta de un Director con 24.000 R. Von. de renta: dos redactores, D. José de Nieva y D. Eustaquio Sedano, con los títulos de Redactor $1^{\circ}$ y $2^{\circ}$ : y dos oficiales, D. Eugenio Amor y D. Manuel de Laraviedra, con los títulos de oficial $1^{\circ}$ y $2^{\circ}$.

D. José de Nieva, redactor $1^{\circ}$, goza de $14.000 \mathrm{RV}^{\text {on }}$. de sueldo, y tiene otros 6.000 por la comisión de reunir, en obra separada que se imprime en la Ymprenta Real, todos los decretos y Reales órdenes; sabe poco o nada; es poco trabajador, y casi inútil para la Gaceta; en cuanto a opinión, aunque es tan nulo como en todo, le creo más adicto a la opinión colateral que a la directa.

Don Eustaquio Sedano, redactor $2^{\circ}$, tiene $12.000 \mathrm{RV}^{\text {on }}$. de sueldo; es un sacerdote anciano y respetable. Si sus conocimientos no son muy estensos, es celoso, y trabaja mucho en lo que se pone a su cargo. Es muy útil en el ramo de anuncios y en la traducción de papeles franceses. Sus opiniones son las del gobierno.

Don Eugenio Amor, oficial $1^{\circ}$, tiene $10.000 \mathrm{RV}^{\text {on }}$. de sueldo. Apostólico furibundo, y por consiguiente hombre ignorantísimo. $\mathrm{Ni}$

3. Sobre los libros que introdujo, véase Juretschke, op. cit., pp. 173-75. 
aun escribir sabe. Sólo se emplea en hacer traducciones pésimas del francés.

D. Manuel Laraviedra, oficial $2^{\circ}$, tiene $8.000 \mathrm{RV}^{\text {on }}$. de sueldo, $\mathrm{y}$ además 3.000 por el trabajo de ir diariamente a las secretarías del despacho a recibir órdenes y recoger noticias. Es el más instruido, inteligente y celoso de toda la oficina, y le recomiendo a V.E., porque le creo el más adicto al gobierno, y capaz de sevir con utilidad en ocupación más elevada.

Tenemos además un porteo muy mal dotado, pues sólo tiene 9 $\mathrm{RV}^{\mathrm{on}}$. diarios, $\mathrm{y}$ se le ha quitado en las últimas economías un egemplar de la Gaceta que se le daba, y que el pobre beneficiaba como podía. Recomiendo a V.E. si es posible, aumentar un poco su sueldo para que pueda vivir más cercano al centro de la villa, pues su corto haber le obliga a habitar en un barrio bajo, y no lo tengo a mis órdenes todas las veces que lo necesito.

Yo, en mi corto entender, aconsejaría que a los Sres. Nieva y Amor se les acomodase en otra parte donde pudiesen ser más útiles y cobrasen algo más de sueldo, de modo que pareciese ascenso; y sus destinos se reemplazasen con hombres más al caso, dando al Sr. Sedano el ascenso de redactor $1^{0}$ y al Sr. Laraviedra el de redactor $2^{\circ}$; y los que entrasen de nuevo fuesen oficiales. (Al margen:) Olvidóseme decir que el Sr. Nieva está condecorado con la Cruz de Carlos III.

$Y$ pues estamos en época de quitar trabas diré a V.E. una que hay en esta oficina, y que me parece muy inútil.

Los escritores de obras o periodicos quieren insertar en la Gaceta sus prospectos: mas esto no puede hacerse, en virtud de Rl. orden, sino por decisión de la Secretaría del Fomento, que ha sucedido a la de Estado en la Inspección de la Gaceta.

Así el autor tiene que presentar un memorial a Fomento, y decretado, presentarlo en esta dirección, donde se le da otro decreto para que su prospecto se inserte. A mí me parece inútil todo esto. Como el director ha de ser siempre un hombre de la confianza del gobierno,me parece que puede dejarse a su arbitrio dar o negar la licencia para la inserción, salvo el derecho de reclamación si abusa, y con la advertencia de que si el libro o el periódico a que se refiere el prospecto trata de materias religiosas o eclesiásticas, haya de presentar con el prospecto el permiso de la autoridad eclesiástica para su publicación; pues V.E. sabe que estos prospectos no están sugetos a censura, y en materias religiosas es menester proceder con esta cautela, y más en el día. Ultimamente deben seguir pagando los autores la obvención de uso en la oficina del cierre de la Gaceta, que creo que son tres duros. Esta obvención es justa, pues la inserción del prospecto aumenta el trabajo de aquellos oficinistas.

Es cuanto por ahora me ocurre que decir en esta materia.

Dios guarde a V.E. muchos años. Madrid 30 de octubre de 1833.

Exmo. Señor (Firmado:) Alberto Lista.

Este escrito va dirigido al Exmo. Sr. Secretario de Estado y del Despacho del Fomento General del Reino. En el margen del último párrafo exten- 
so sobre los prospectos, la persona que informó el asunto de Lista ha escrito a mano: "Por lo tocante a esta indicac". póngase al despacho".

El resumen de este texto dice: "1833. Informe de Dn. Alberto Lista dando cuenta del estado del personal de la Gaceta".

Probablemente es el Secretario de Estado y del Despacho del Fomento General del Reino quien hizo las dos anotaciones al margen del escrito de Lista.

Manuel Chaves se refirió en su excelente biografía sobre Lista, antes citada, a su labor en este órgano oficial que era la Gaceta de Madrid (op. cit. pp.39-40). Así mismo lo hace Juretschke en su monografía también citada (op. cit. pp.150-59).

Según este último autor, Lista procura ejercer su magisterio a través de la Gaceta, formar y educar (p. 155).

Pérez de Guzmán en su Bosquejo histórico-documental de la "Gaceta de Madrid", Madrid, 1902, p. 164, comenta que Lista dio al periódico un giro más literario, sin fortuna. Pero Juretschke destaca los escasos artículos literarios o culturales que se publicaron durante su dirección del periódico, limitándose a cortas reseñas de amigos (pp. 155-56).

Según Chaves, cuando Cea Bermúdez fue a ver a Lista a Bayona, éste le pidió le concediera la dirección del periódico (ibíd. p. 154).

Juretschke comenta las consignas que Lista tuvo que difundir, que originaron protestas entre los jóvenes que le leían (p. 156).

El crítico alemán, con cuyas teorías acerca de la ideología de Lista en esta época estamos en desacuerdo - como demostraremos ampliamente en el libro que preparamos sobre el tema-, recoge que el propio autor se defendió de que le hicieran responsable del contenido de la Gaceta, aunque Martín Villa señala que Lista determinó la propaganda en la cuestión sucesoria (p. 157, op. cit.).

Lista reunió un equipo de redactores muy notable, y al decir de Juretschke fue mucho más que el director de un periódico oficial - desde enero de 1833 hasta aproximadamente junio de 1837 - para intervenir en asuntos de alta política (p. 158).

Los documentos que hemos presentado, en espera de un análisis más pormenorizado que aquí no podemos hacer por motivos de espacio, son de tres tipos: el nombramiento como director de la Gaceta, la petición de libros prohibidos de su propiedad y la reestructuración que realizó en la redacción del periódico nada más ponerse a su frente.

El primer documento ha sido comentado por Chaves y por Juretschke, y recoge simplemente los trámites de su nombramiento, y los motivos por 
los que Pedro de la Hoz renunció al cargo.

El segundo paquete de documentos nos revela hasta qué punto era difícil y penoso, en esta época, el hacer penetrar libros prohibidos en el país, aún con el consentimiento de los cargos oficiales, y que la Iglesia era quien ponía las trabas más importantes a este tema. Aunque Lista consiguió su objetivo.

El tercer aspecto que se estudia en estos documentos nos revela a un Alberto Lista emprendedor, que realiza un informe confidencial en el que denuncia sin empacho alguno a los redactores inútiles, consiguiendo un nuevo vuelo para la publicación al remozarla y rejuvenecerla. Nótese que Lista es consciente de que su labor en el periódico consiste simplemente en estar al servicio del gobierno, y no pueden imputársele las ideas de aquél, como hará Juretschke a propósito de Mendizábal, con quien, pese a lo que diga el crítico alemán, no está comprobada su colaboración.

Una anotación final, respecto a este tercer aspecto como director de la Gaceta: Lista es implacable con los inútiles en su informe, pero tiene una llamada de humanitarismo con los humildes que desempeñan bien su trabajo, como se demuestra al referirse al portero mal pagado. Es un rasgo de su humanidad constante.

Para una mayor información remitimos al lector interesado a nuestro libro de próxima publicación Ideología y Literatura en Alberto Lista. 